\title{
RED BLOOD CELL IMAGE ENHANCEMENT TECHNIQUES FOR CELLS WITH OVERLAPPING CONDITION
}

\author{
L. Oussama ${ }^{1}$, M. M. A. Jamil ${ }^{1, *}$, W. M. Hafizah ${ }^{1}$ and R. Ambar ${ }^{2}$ \\ ${ }^{1}$ Biomedical Modelling and Simulation (BIOMEMS) Research Group, Department of \\ Electronic Engineering, Faculty of Electrical and Electronic Engineering, Universiti Tun \\ Hussein Onn Malaysia, Batu Pahat, Johor, Malaysia \\ ${ }^{2}$ Embedded Computing System (EmbCoS) Research Group, Department of Computer \\ Engineering, Faculty of Electrical and Electronic Engineering, Universiti Tun Hussein Onn \\ Malaysia, Batu Pahat, Johor, Malaysia \\ Published online: 05 October 2017
}

\begin{abstract}
This paper proposes an automatic algorithm consists of techniques that can separate overlapping $\mathrm{RBC}$ for enhancing $\mathrm{RBC}$ image to improve counting precision. The proposed algorithm is able to automatically count RBC of colour blood smear images based on the quality or challenging conditions of the images such as poor illumination of blood smear and most importantly overlapping RBC. The algorithm comprises of two RBC segmentation that can be selected based on the image quality, circle mask technique and grayscale blood smear image processing. Detail explanations including experimental results that show the effectiveness of the proposed techniques are described in this paper. The proposed algorithm has been successfully detect and separate agglomerate RBC with 96\% accuracy. Algorithm validation was verified with $\mathrm{RBC}$ distribution area and confusion matrix.
\end{abstract}

Author Correspondence, e-mail: mahadi@uthm.edu.my

doi: http://dx.doi.org/10.4314/jfas.v9i4s.35 
Keywords: red blood cell (RBC); complete blood count (CBC); automated segmentation; overlap RBC.

\section{INTRODUCTION}

A complete blood count (CBC) is a common test performed in pathology laboratories for diagnosing diseases and helps to assess many symptoms of diseases [1]. Furthermore, CBC test results are able to reflect the amount of blood volume. A CBC test involves three evaluations; white blood cell (WBC), platelet and red blood cell (RBC) evaluations. RBC count is one of the tests in RBC evaluation that can detect diseases and other condition that affecting RBC [2]. The other conditions which an RBC count may be conducted are hereditary anemias such as thalassemia, drug-induced immune hemolytic anemia, idiopathic autoimmune hemolytic anemia, hemolytic anemia due to G6PD and Waldenström macroglobulinemia [3].

For most diseases described above, clinical examinations are required for advance medical examination decision [3]. In addition, further medical examination requires more precious early clinical examination results which compel to use software for analysis and measurement especially in applications based on detecting and extracting image features. The algorithms developed using the software is the gist for analysis to measure features and detect it exactly. For this purpose, automatic examination using dedicated devices are widely used especially for complicated medical type of images to extract region of interest (ROI) and compute necessary dimensions like surface and area covered by objects.

In clinical examination, a $\mathrm{CBC}$ test is usually executed using sophisticated automated cell counter device which provides rapid and precise result. Some of these devices can process more than 100 samples per hour. However, a major drawback of these devices, especially impedance based devices is it can only check the volume and quantity of particles, and might not be able to properly differentiate tiny platelet clumps and nucleated red blood cells [4]. Platelets clumps may be misclassified as leukocytes or erythrocytes and nucleated red blood cells can be misclassified as leukocytes. On the other hand, some devices utilize flagging system to help identify abnormal cell counting [5]. The devices are pre-programmed to produce certain flags according to the internal algorithm such as nucleated RBC. 


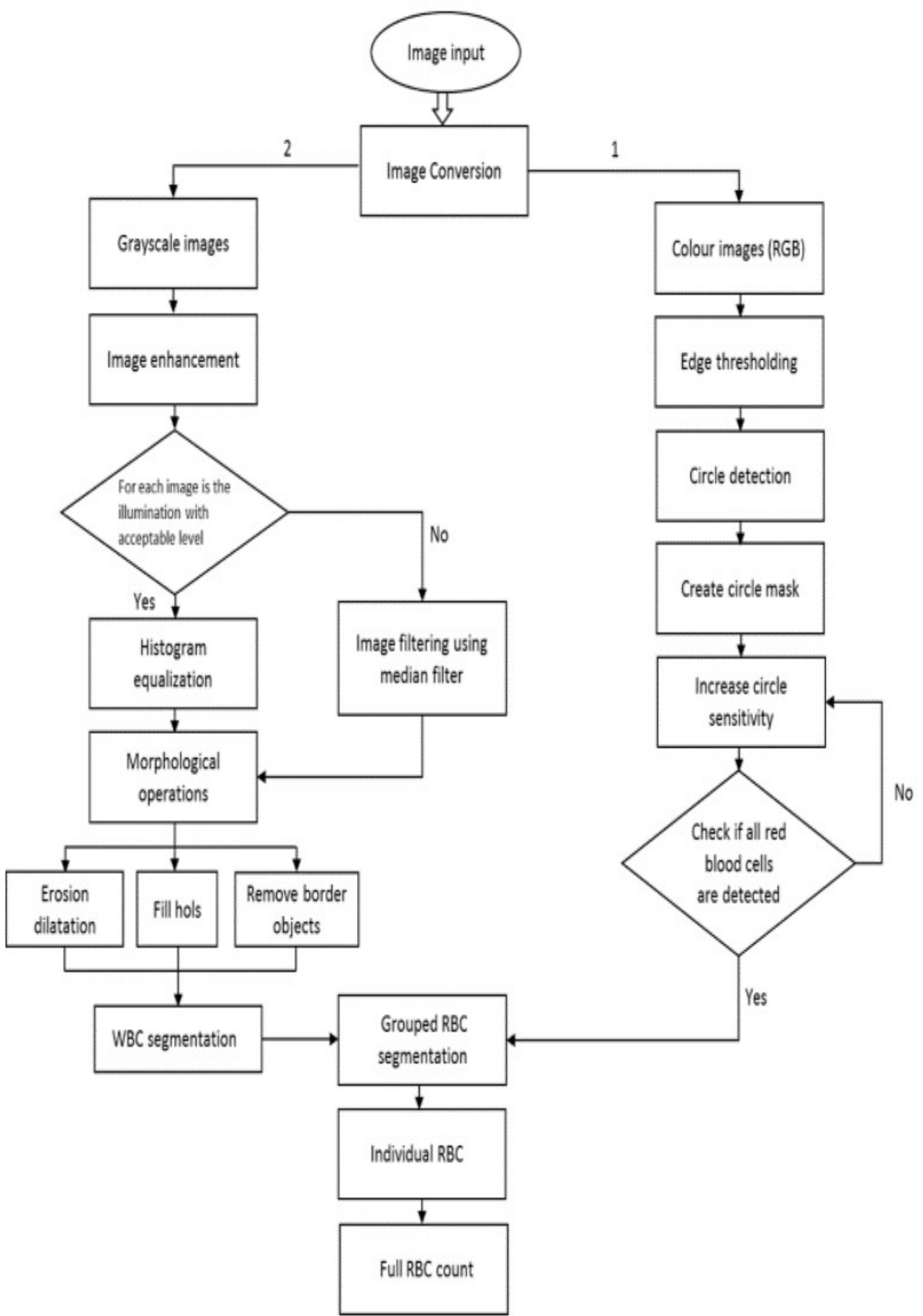

Fig.1. Flowchart of the proposed algorithm for RBC image enhancement technique for cells with overlapping condition 
Flag and flag for disparity between two blood cell counts. However, these devices are unable to detect overlapping $\mathrm{RBC}$ and also produce false value of cell counting in overlapping condition [6]. Therefore, referring to the above drawbacks of existing devices, an automatic algorithm that can detect RBC and separate overlapping RBC is necessary.

In our previous work, RBC classification in blood smear image was conducted [7]. However, the work only classified the normal, abnormal and overlapping cells without separating the agglomerates RBC. In this paper, an automatic technique of blood cell examination that is able to detect $\mathrm{RBC}$ and separate overlapping $\mathrm{RBC}$ into single $\mathrm{RBC}$ is presented. In the proposed technique, the algorithm that comprises of two RBC segmentation techniques is proposed. The new algorithm will help to derive more accurate results, less time consuming and avoid any misclassification of illness type.

\section{THE PROPOSED ALGORITHM}

Fig. 1 shows the flowchart of the proposed automatic algorithm to detect RBC and separate overlapping $\mathrm{RBC}$ into single $\mathrm{RBC}$, which consists of two RBC segmentation techniques.

The algorithm starts when a colour blood smear image is fed into the algorithm, where image conversion will be carried out to determine the quality of the image. If the quality of the input image is good, the image will be automatically processed by circle mask technique which is labelled with (1) in Fig. 1. On the other hand, if the quality of the image is determined low, it will be processed using pre-processing first then RBC extraction labelled with (2) in Fig. 1. In this paper, we will discuss only about the two RBC segmentation techniques, (1) and (2).

The circle mask technique (1) uses edge thresholding to detect RBC borders and circle masks to identify individual $\mathrm{RBC}$ in images. This method is accurate for detecting single $\mathrm{RBC}$ in good quality images that have less overlapping RBC condition.

The second technique (2) uses pre-processing technique to produce images with clear and shaped features. This technique is divided into three subsystems; pre-processing, automatic $\mathrm{RBC}$ extraction and overlapping $\mathrm{RBC}$ segmentation. In pre-processing, image is prepared for processing steps which include grayscale conversion and image enhancement. Then, automatic RBC extraction is the step that detects and counts single cells only. Whereas, overlapping $\mathrm{RBC}$ segmentation process enable the separation of agglomerate RBC which includes morphological operation to diminish non-cell objects for identifying single RBC. The second method is suitable for detecting single $\mathrm{RBC}$ in poor quality images that have many overlapping RBC. 
Then, both techniques utilize a controlled watershed technique for segmentation of RBC inner and outer marker (IOM) to avoid over-segmentation issue. Furthermore, city block distance technique is used to measure segmented image features. As the final step, boundary chain code technique has been implemented to encode the results of image separation. Detail explanations for both techniques are described in the next subsections.

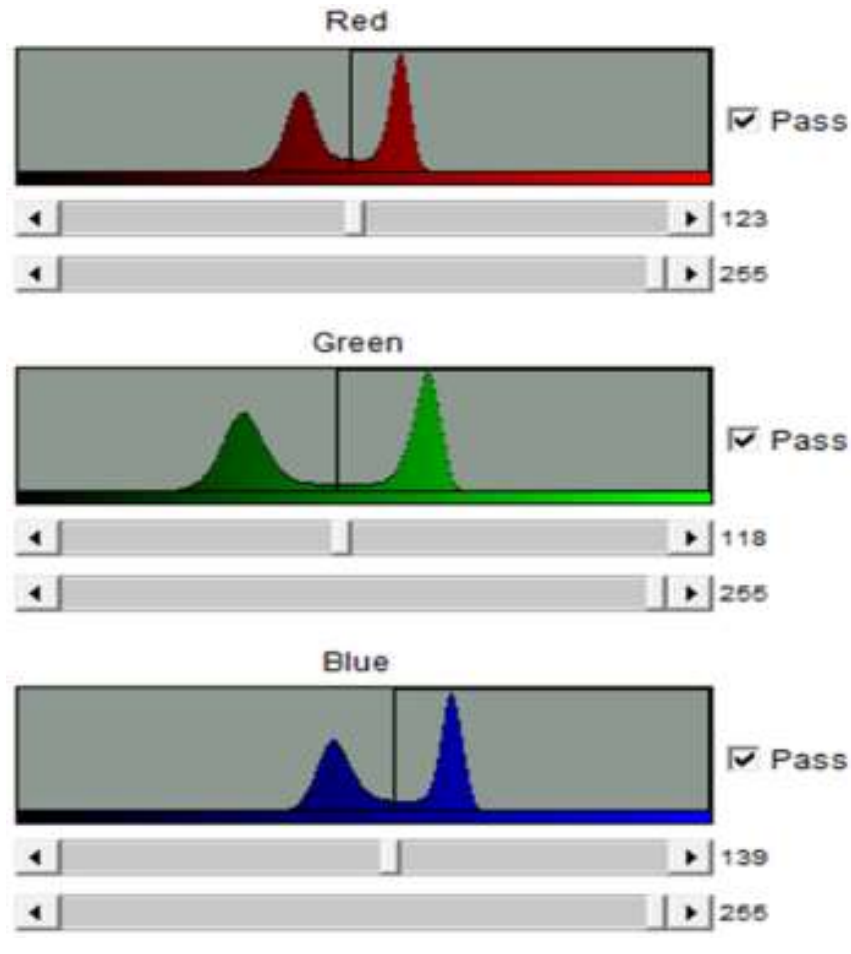

(a)

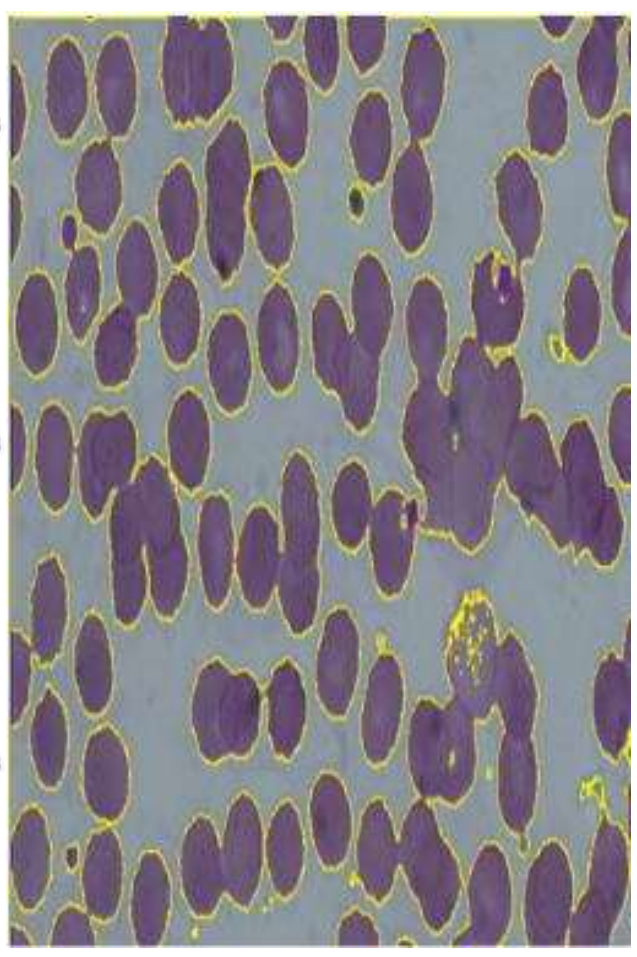

(b)

Fig.2. Edge detection using colour thresholding

\section{CIRCLE MASK TECHNIQUE}

This method is suitable for good quality images that have less overlapping RBC condition. As explained previously, colour thresholding technique utilizes edge thresholding to detect RBC borders. In order to threshold an RGB image, threshold value for each colour component needs to be specified first. Here, segmentation of overlapping RBC using this technique is described.

Fig. 2 shows the method to modify three colours (red, green and blue) threshold values using adjustment sliders. As shown in Fig. 2(a), threshold value for red is 123 pixel, 118 pixel for green and 139 pixel for blue. The optimum values for affinity and homogeneity thresholds were found by changing them and watching the changes of each colour. Fig. 2(b) shows the result of a colour blood smear image after changing the threshold values based on Fig. 2(a). 
As seen in the image, edge thresholding can differentiate in contrast between background and single RBC where circle RBC edges are shown clearly. The circle edges are detected to create circle mask on each RBC. However, the image shows that the overlapping RBC still unseparated. This can be done by increasing the colour sensitivity and centroid technique.

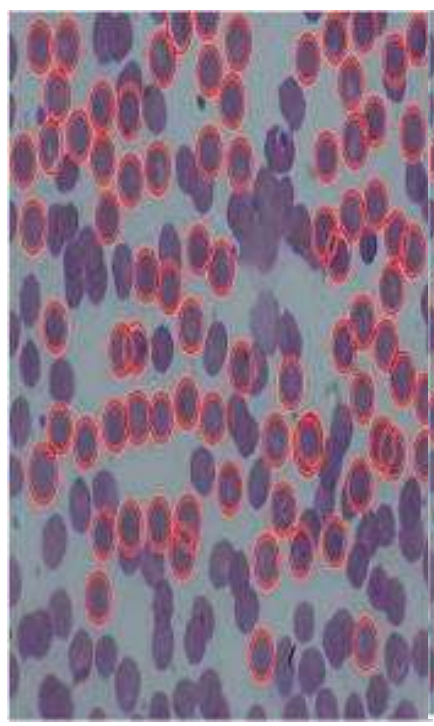

(a)

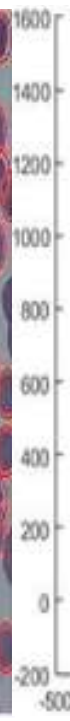

(b)

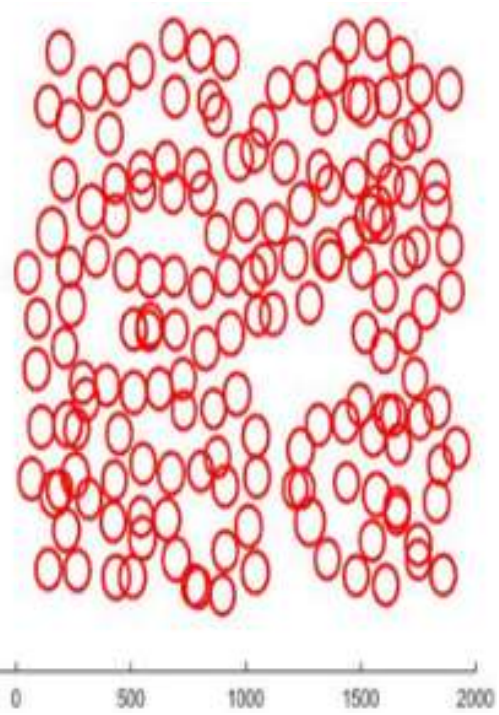

(c)

Fig.3. RBC detection using colour thresholding with circle mark (a) Single RBC detected (b) More single RBC detected after increasing circle mask sensitivity (c) RBC separated from the background using XOR operation

Fig. 3 shows example of colour blood smear image treated with this technique. Fig. 3(a) shows that the technique detected several single RBC. Circle masks are created to show the detected RBC. Then, Fig. 3(b) shows an increase of detected RBC even with high overlapping RBC. This is achieved after increasing circle sensitivity and using centroid technique. Fig. 3(c) shows RBC separated from the background using XOR operation to make counting easier. 


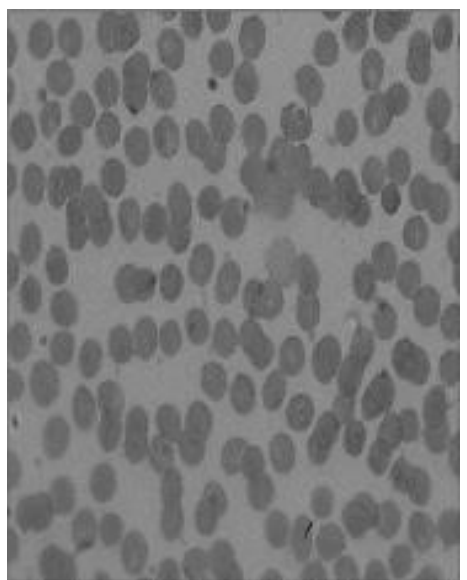

(a)

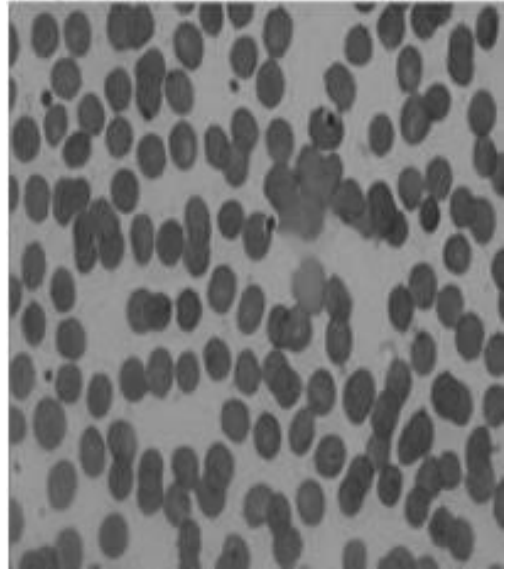

(b)

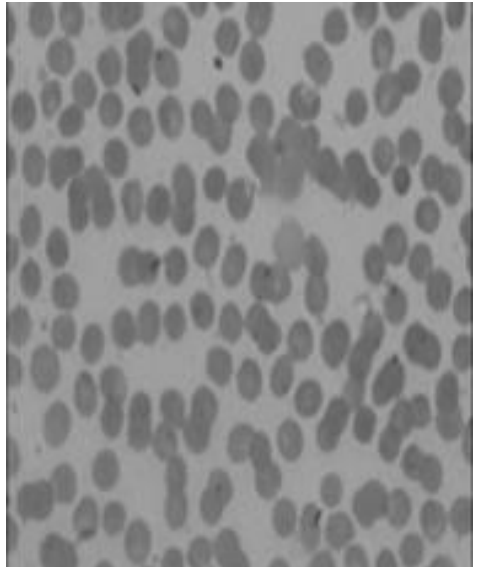

(c)

Fig.4. RGB blood image split into three planes (a) red (b) green (c) blue

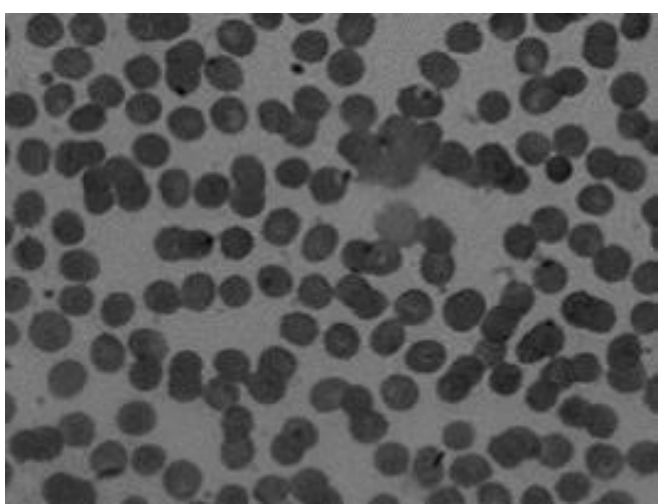

(a)

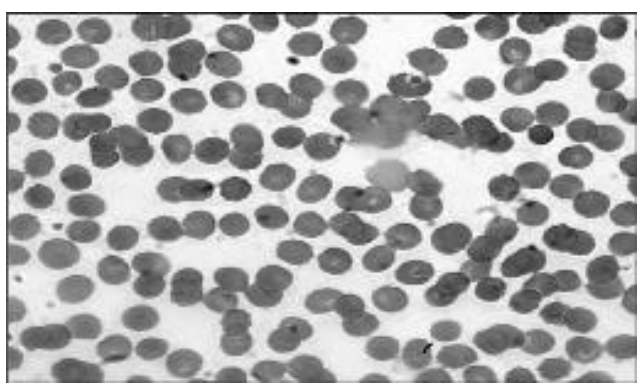

(c)

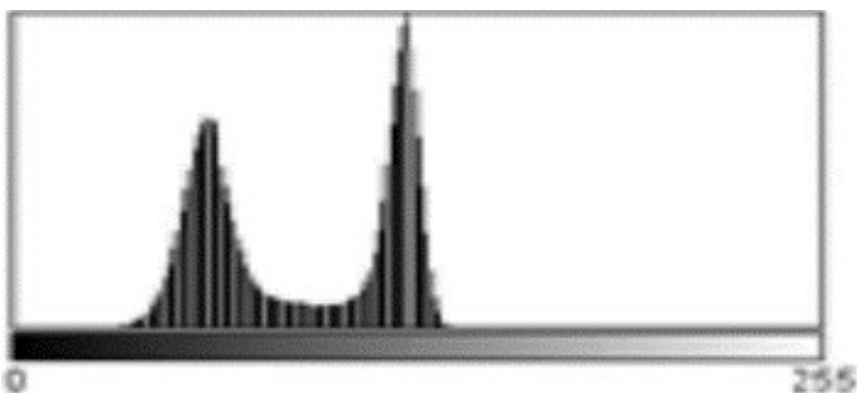

Count 2949120 Mean: 91.893 stober: $304: 3$

$\operatorname{Max} 179$

Mode: $124(127392)$

(b)

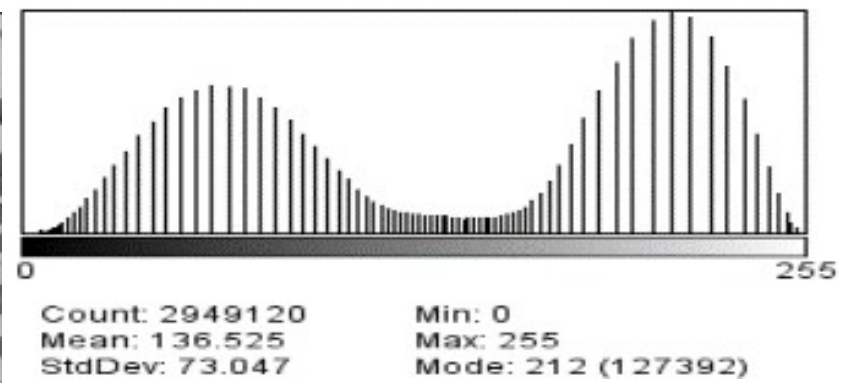

(d)

Fig.5. Blood image with histogram before and after image enhancement technique (a) Original image (b) Image histogram (c) Equalized image (d) Histogram of equalized image

\section{GRAYSCALE IMAGE PROCESSING}

This method is suitable for images that poor image quality contain and high quantity of overlapping RBC or which need to be pre-processed to make image features clear and shaped. 
There are three subsystems involved; pre-processing, automatic RBC extraction and overlapping RBC segmentation as explained previously.

\subsection{Pre-Processing}

Pre-processing stage consists of grayscale conversion, image enhancement and morphological operation.

\subsubsection{Grayscale Conversion}

In this process, the red and blue channel of the input image is eliminated, while green channel is kept. This is due to the fact that blood cells have more contrast in green channel of RGB colour plane as shown in Fig. 4.

\subsubsection{Image Enhancement}

After grayscale conversion, the image undergoes image enhancement process. Image enhancement is the process of improving image quality and enhancing the appearance of an image or a subset of the image for better contrast or visualization of certain features and to facilitate subsequently more accurate image analysis. Usually, for image enhancement, some of the techniques that could be used are: point processing, histogram based techniques or mask processing in spatial domain or (high, low) filter in frequency domain [8]. In this work, spatial domain technique is implemented because enhancement operations are carried out on the whole image or on local region selected on the basis of image statistics such as histogram equalization, image averaging sharpening of important features such as contours or edges and nonlinear filtering (median filtering).

Fig. 5 shows the result of image enhancement technique with histogram of equalized image. Fig. 5(a) shows the original image of RBC. Fig. 5(b) shows the histogram of the original image. Fig. 5(c) shows the equalized original image. Fig. 5(d) shows the histogram of the equalized image which easily separate between objects and background.

\subsubsection{Morphological Operation}

The enhanced image is treated with morphological operation. Morphological operation is based on a strong mathematical concept, which is used to change structure, size of blood image, remove border objects and fill the holes. Morphological operation involves binary erosion, dilation, opening and closing. The technique can also be extended to be used for grayscale image. Morphological opening remove, break and diminished the connection or objects which not contain the structure elements [9]. In contrary, morphological closing functions to join, fill and build connection and objects in the image. However, both of opening and closing operation have similar task which is smoothen the object contours. 
Morphological operation has been implemented in the proposed algorithm to remove border objects and fill holes as shown in Fig. 6.

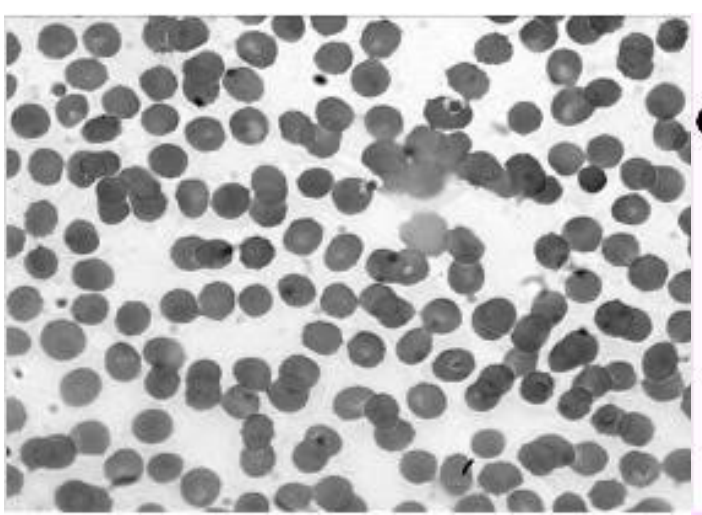

(a)

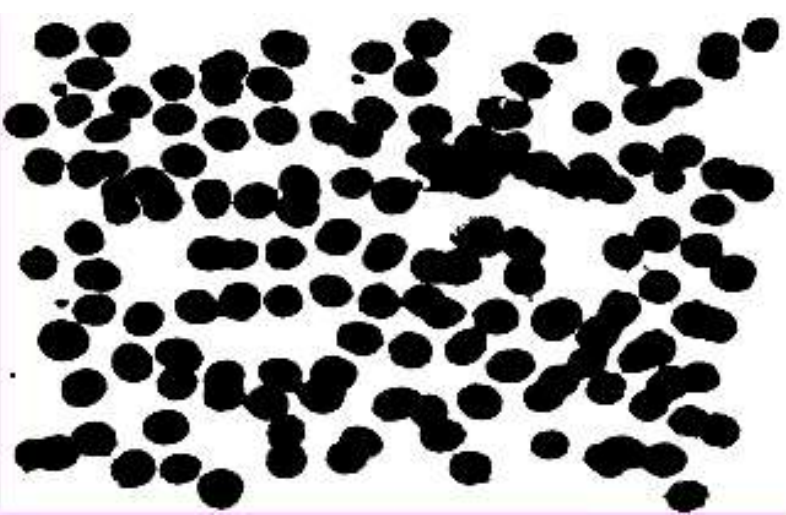

(b)

Fig.6. Morphological operation (a) Before morphological operation (b) After morphological operation

Table 1. Confusion matrix

\begin{tabular}{|c|c|c|}
\hline & A Cell is Detected & No Cell is Detected \\
\hline $\begin{array}{l}\text { A cell } \\
\text { exists }\end{array}$ & $\begin{array}{l}\text { a. The number of truly segmented } \\
\text { cells by the algorithm }\end{array}$ & $\begin{array}{l}\text { b. The number of true cells that are not } \\
\text { segmented by the algorithm }\end{array}$ \\
\hline $\begin{array}{l}\text { No cell } \\
\text { exists }\end{array}$ & $\begin{array}{l}\text { c. The number of falsely segmented } \\
\text { cells by the algorithm }\end{array}$ & $\begin{array}{l}\text { d. The number of cells on the image where } \\
\text { no cell segmented and algorithm do not } \\
\text { consider as segmented cells }\end{array}$ \\
\hline
\end{tabular}

Table 2. Algorithm evaluation based on six images

\begin{tabular}{lcccccc}
\hline & $\begin{array}{c}\text { Correctly } \\
\text { Detected Cells }\end{array}$ & $\begin{array}{c}\text { Cells Not } \\
\text { Detected }\end{array}$ & $\begin{array}{c}\text { Falsely } \\
\text { Detected Cells }\end{array}$ & Accuracy & Recall & Precision \\
\hline Image 1 & 108 & $(\mathbf{b})$ & $(\mathbf{c})$ & & & \\
Image 2 & 124 & 13 & 6 & 95.27 & 89.25 & 94.73 \\
Image 3 & 98 & 5 & 8 & 94.16 & 96.12 & 93.93 \\
Image 4 & 130 & 15 & 4 & 96.58 & 86.72 & 96.07 \\
Image 5 & 128 & 3 & 3 & 97.79 & 97.74 & 97.74 \\
Image 6 & 134 & 2 & 8 & 94.2 & 98.46 & 94.11 \\
Average & 121 & 0 & 5 & 96.4 & 100 & 96.4 \\
\hline
\end{tabular}




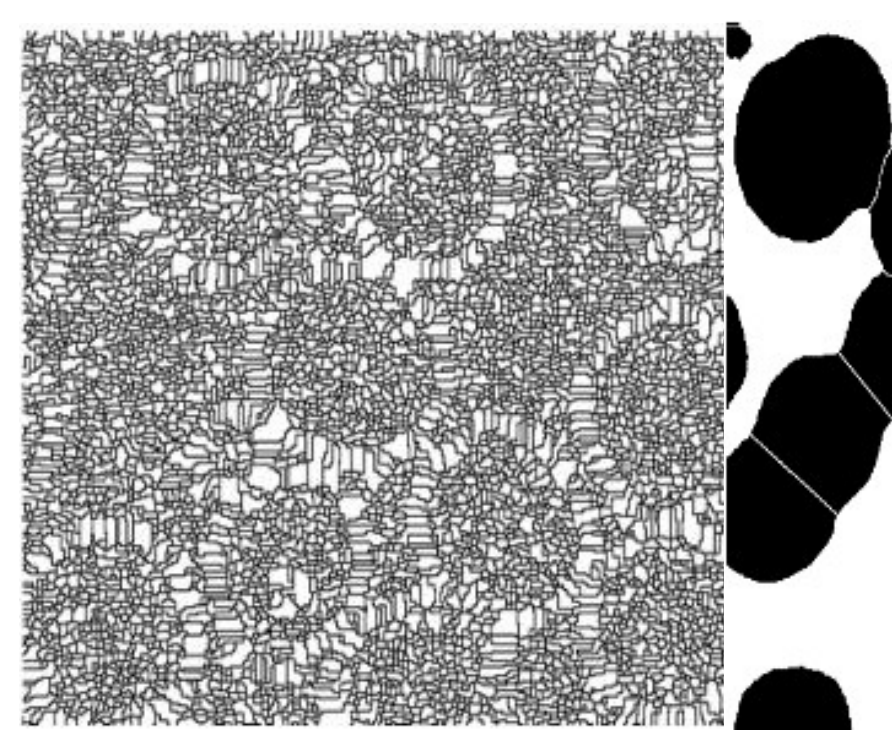

(a)

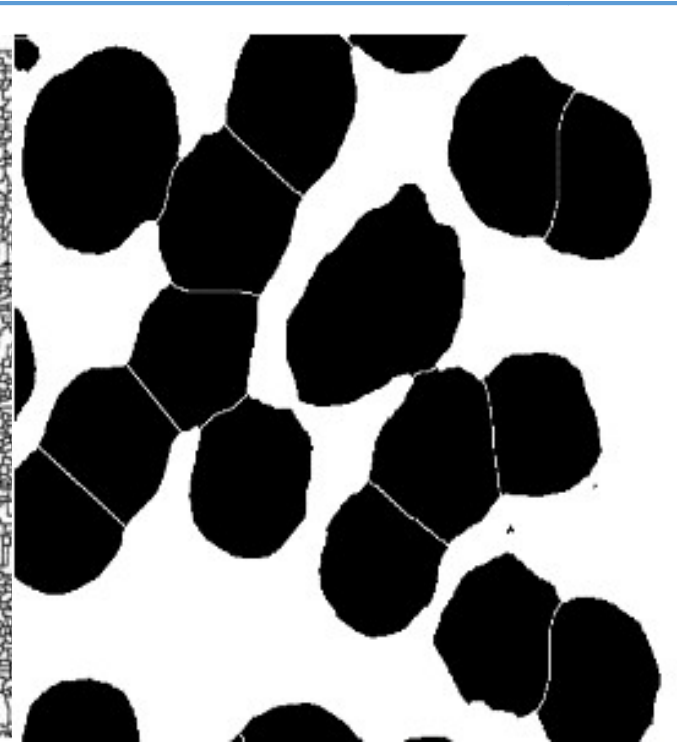

(b)

Fig.7. (a) Over-segmentation issue and, (b) solving over-segmentation issue using control markers

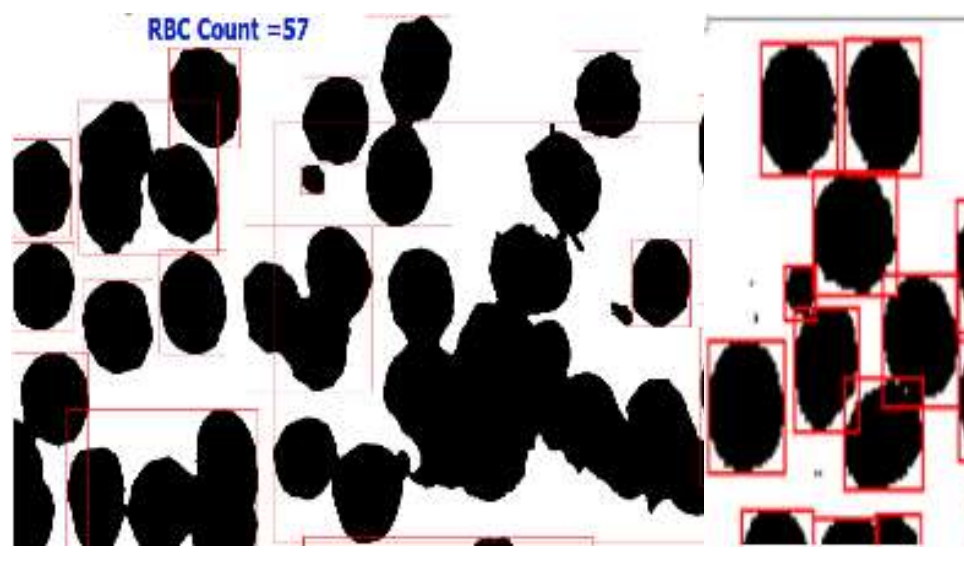

(a)

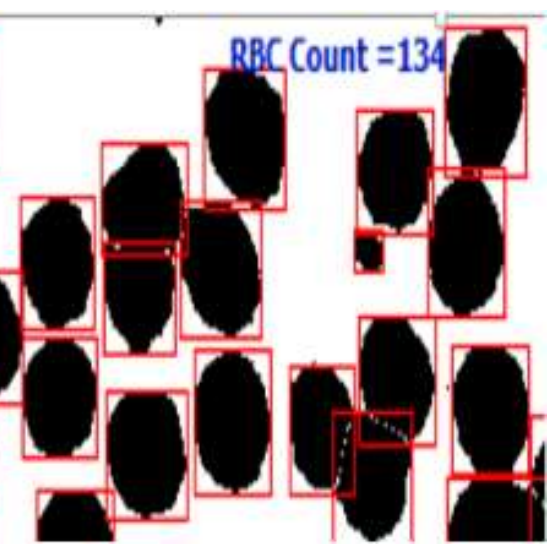

(b)

Fig.8. RBC count before and after solving over-segmentation process (a) Before segmentation (b) After segmentation 

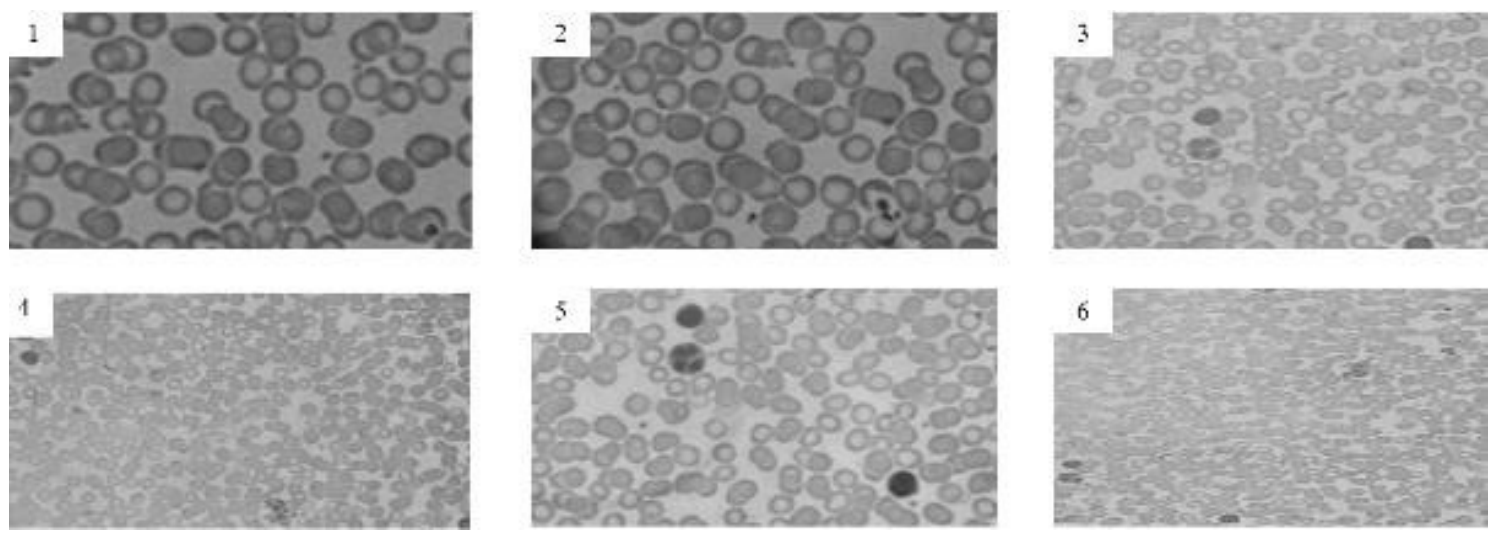

Fig.9. Pattern images chosen randomly from blood smear images
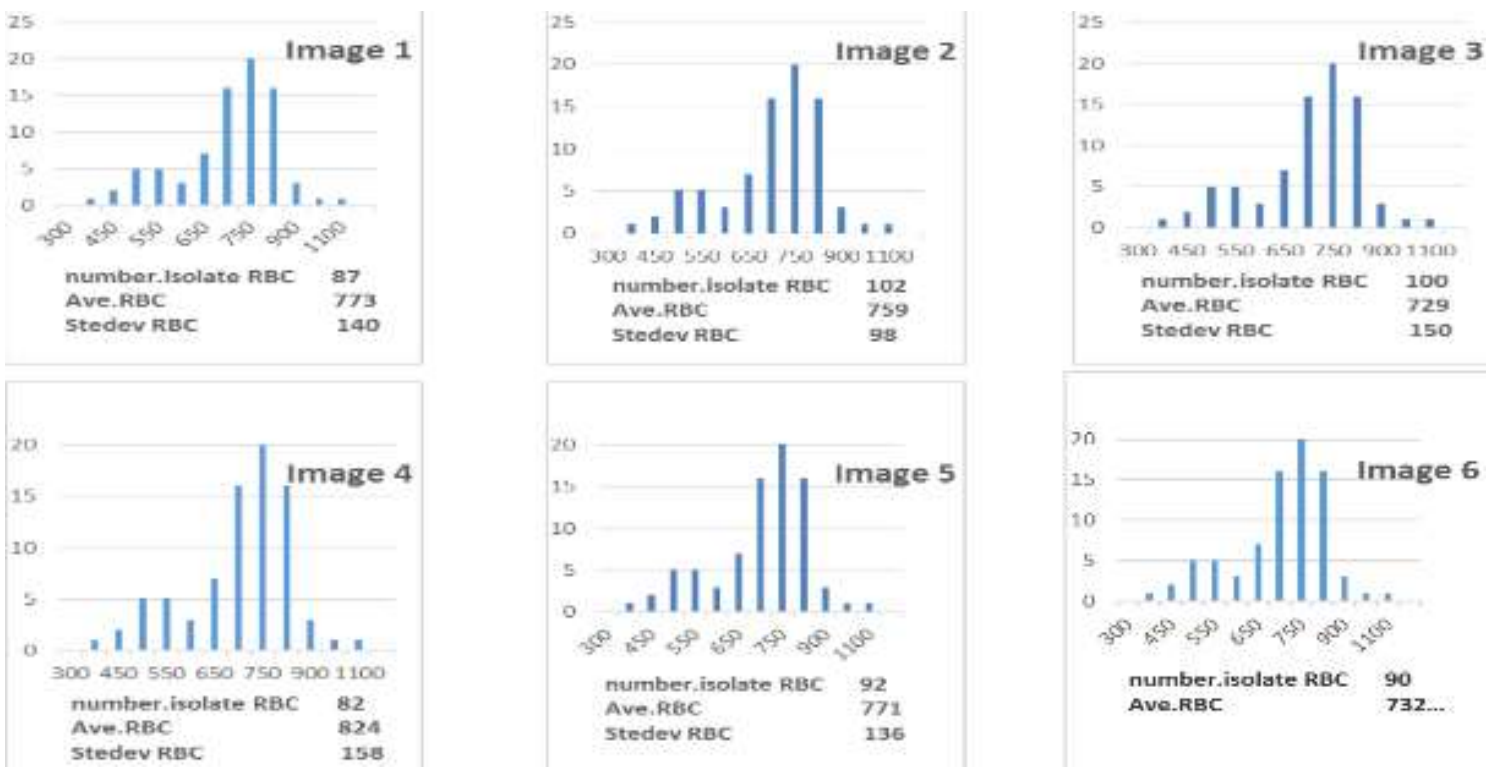

Fig.10. Number of isolated RBC and area of distribution for six images chosen randomly in

Fig. 9

\section{OVERLAPPING RBC SEGMENTATION}

In overlapping $\mathrm{RBC}$ segmentation process, a blood image is divided into region of interest (ROI) with homogony regions over segmentation issue is controlled by IOM implementing on watershed technique.

Fig. 7(a) shows an image of over-segmentation issue. Fig. 7(b) shows the control of segmentation using IOM in image. Fig. 8 shows RBC count before and after solving over-segmentation issue by separating overlapping RBC.

As can be seen in Fig. 8, before segmentation, each overlapping RBC is counted as a single $\mathrm{RBC}$. This is due to indifferent contrast between background and objects. Therefore, no sign for existing cell is detected. However, using the proposed algorithm, for each overlapping 
$\mathrm{RBC}$, border separation between cells are created after segmentation process as shown in Fig. 8. The figure shows that before segmentation, only $57 \mathrm{RBC}$ were counted. But, after overlapping RBC segmentation, an additional $77 \mathrm{RBC}$ were counted successfully.

\section{DISCUSSION}

\subsection{Algorithm Validation}

Average area of the separated RBC is used to validate the proposed algorithm. It is a robust technique that can validate and confirm the correct results of RBC clustering considering the overlapping pixels between the minimum two RBC [10].

Fig. 9 shows six different patterns of RBC images taken randomly. Fig. 10 shows the number of isolated RBC and area of distribution for six images shown in Fig. 9. For each blood image, there is sufficient number of isolated RBC. The controlling of the area of RBC was validated by counting the separated red blood cells a normal distribution approximately is $767 \pm 142$ pixels of $\mathrm{RBC}$, which implies $15.7 \pm 1.3$ pixels of $\mathrm{RBC}$ radius and the area are $57 \sim 108$ pixels separated RBC which is enough number to calculate average $\mathrm{RBC}$ area. The best distribution of the area is the distribution follows the normal distribution with less variation [10].

\subsection{Algorithm Performance}

In preparation for describing the performance of the proposed algorithm, a brief review of performance measurements in pattern recognition is presented. The aim in pattern recognition is to automatically find particular patterns in a population with different patterns cases [11]. The outcomes of such process is true or false prediction. In this work, the aim is segmentation of RBC. Table 1 shows the confusion matrix for the scenarios that can happen in separating $\mathrm{RBC}$ in an image.

Performance of the algorithm such as accuracy, recall and precision can be measured based on the confusion matrix. Below are details of each perimeter:

- Accuracy which is the percentage of correct predicted from the total number that are tested [12]. Accuracy is defined as:

Accuracy $=\frac{a+d}{a+b+c+d}$

- Recall is the percentage of the number of the cells detected correctly from the total of actual number of cells.

Recall $=\frac{\mathrm{a}}{\mathrm{a}+\mathrm{b}}$

- Precision is the percentage of the number of correctly detected cells to the sum of correctly and falsely detected cells. 
Precision $=\frac{\mathrm{a}}{\mathrm{a}+\mathrm{b}}$

Table 2 shows the results of the three perimeters (accuracy, recall and precision) for six different patterns of RBC images taken randomly. The table also shows the average area values for the components in Table 1 (components (a), (b) and (c)). As shown in Table 2, the average precision and recall values detected by the proposed algorithm is $95.49 \%$ and $94.71 \%$ respectively where the errors of counting are less than $5 \%$.

Basically, apart from using automatic RBC counter devices, traditionally ways of RBC count can be done using manual cell counting which is performed using microscopes and hemocytometer. The drawback of manual counting is that the precision is highly dependent on the operator which may prone to error.

\section{CONCLUSION}

This paper described an automatic algorithm for RBC image enhancement techniques for cells with overlapping conditions. The proposed automatic algorithm is consists of two techniques that can separate overlapping RBC to improve counting precision for any good and bad quality colour blood smear images. The proposed algorithm has been tested on 46 images of blood sample including bad illumination images and low contrast with high overlapping condition. Many methods that have been implemented for RBC separation show bad results in the case of overlapping between RBC and bad illumination. The algorithm developed in this work demonstrated average recall and precision values of approximately $95 \%$. Furthermore, the proposed method is possible to identify and separate RBC with an average accuracy of $96 \%$. The performance of the proposed method show significantly correct results in all of the cases and an approximate count of red blood cells. In this work, two robust methods for automatic segmentation has been proposed in order to provide automated procedure for medical analysis. The results obtained show that the proposed method can provide an innovative way of identifying $\mathrm{RBC}$ and separating the overlapping $\mathrm{RBC}$. The main advantage of developed algorithm is the ability to separate automatically RBC in high overlapping condition and poor illumination. The procedure has been executed in Java and MATLAB environment. 


\section{ACKNOWLEDGEMENTS}

The authors would like to thank Universiti Tun Hussein Onn Malaysia (UTHM) for financial support through (ORICC) for this project under graduate incentive research grant vote number U166.

\section{REFERENCES}

[1] Rao L V, Ekberg B A, Connor D, Jakubiak F, Vallaro G M, Snyder M. Evaluation of a new point of care automated complete blood count (CBC) analyzer in various clinical settings. Clinica Chimica Acta, 2008, 389(1):120-125

[2] Carroll C, Marsden P, Soden P, Naylor E, New J, Dornan T. Involving users in the design and usability evaluation of a clinical decision support system. Computer Methods and Programs in Biomedicine, 2002, 69(2):123-135

[3] Vincent J L, Baron J F, Reinhart K, Gattinoni L, Thijs L, Webb A, Meier-Hellmann A, Nollet G, Peres-Bota D, ABC Investigators. Anemia and blood transfusion in critically ill patients. Journal of American Medical Association, 2002, 288(12):1499-1507

[4] Lokwani, D. P. The $\mathrm{ABC}$ of $\mathrm{CBC}$ : Interpretation of complete blood count and histograms. New Delhi: Jaypee Brothers Medical Publishers (P) Ltd., 2013

[5] Weiser M G, Vap L M, Thrall M A. Perspectives and advances in in-clinic laboratory diagnostic capabilities: hematology and clinical chemistry. Veterinary Clinics of North America: Small Animal Practice. 2007, 37(2):221-236

[6] Sakamoto C, Yamane T, Ohta K, Hino M, Tsuda I, Tatsumi N. Automated enumeration of cellular composition in bone marrow aspirate with the CELL-DYN 4000TM automated hematology analyzer. Acta Haematologica, 1999, 101(3):130-134

[7] Tomari R, Zakaria W N, Jamil M M, Nor F M, Fuad N F. Computer aided system for red blood cell classification in blood smear image. Procedia Computer Science, 2014, 42:206-213

[8] Sawant H K, Deore M. A comprehensive review of image enhancement techniques. International Journal of Computer Technology and Electronics Engineering, 2010, 1(2):39-44 [9] Sharif J M, Miswan M F, Ngadi M A, Salam M S, Abdul Jamil M M. Red blood cell segmentation using masking and watershed algorithm: A preliminary study. In IEEE International Conference on Biomedical Engineering, 2012, pp. 258-262

[10] Moon S, Lee S, Kim H, Freitas-Junior L H, Kang M, Ayong L, Hansen M A. An image analysis algorithm for malaria parasite stage classification and viability quantification. Plos One, 2013, 8(4):1-12 
[11] Arslan S, Ozyurek E, Gunduz-Demir C. A color and shape based algorithm for segmentation of white blood cells in peripheral blood and bone marrow images. Cytometry Part A, 2014, 85(6):480-490

[12] Grau V, Mewes AU, Alcaniz M, Kikinis R, Warfield S K. Improved watershed transform for medical image segmentation using prior information. IEEE Transactions on Medical Imaging, 2004, 23(4):447-458

\section{How to cite this article:}

Oussama L, Jamil M M A, Hafizah W M, Ambar R. Red blood cell image enhancement techniques for cells with overlapping condition. J. Fundam. Appl. Sci., 2017, 9(4S), 614-628. 